\title{
Self-Propulsion of Water-Supported Liquid Marbles Filled with Sulfuric Acid
}

\author{
Mark Frenkel, ${ }^{\dagger}$ Leonid Dombrovsky, ${ }^{\ddagger}$ Victor Multanen, $^{\dagger}, \|$ Viktor Danchuk, ${ }^{\perp}$ Irina Legchenkova, ${ }^{\dagger}$ \\ Shraga Shoval, ${ }^{\#}$ Yelena Bormashenko, ${ }^{\dagger}$ Bernard P. Binks, ${ }^{\bigcirc}$ and Edward Bormashenko ${ }^{*} \dagger \odot$ \\ ${ }^{\dagger}$ Chemical Engineering, Biotechnology and Materials Department, Engineering Faculty, Ariel University, P.O.B. 3, 40700, Ariel, \\ Israel \\ ${ }^{\ddagger}$ University of Tyumen, Tyumen, 625003, Russia \\ $\S$ Joint Institute for High Temperatures, Moscow, 111116, Russia \\ "Nanoprobe Lab for Bio- \& Nanotechnology \& Biomimetics, Ohio, College of Engineering, The Ohio State University, Columbus, \\ Ohio 43210-1142, United States \\ ${ }^{\perp}$ Department of Physics, Exact Sciences Faculty, Ariel University, P.O.B. 3, 40700, Ariel, Israel \\ \#Industrial Engineering and Management, Engineering Faculty, Ariel University, P.O.B. 3, 40700, Ariel, Israel \\ OSchool of Mathematics and Physical Sciences, University of Hull, Hull, HU6 7RX, U.K.
}

\section{Supporting Information}

ABSTRACT: Self-propulsion of liquid marbles filled with sulfuric acid and coated with hydrophobic fluorosilica powder on a water surface is reported. The prolonged self-propulsion of marbles occurs over a couple of minutes with a typical velocity of the center of mass of the marble being $v_{\mathrm{cm}} \sim 0.01 \frac{\mathrm{m}}{\mathrm{s}}$. The shell of the marble is not uniform, resulting in the asymmetric absorption of water by a marble, giving rise to the nonuniform thermal field within its volume. The maximum temperature reached at the liquid marble surface was $70{ }^{\circ} \mathrm{C}$. The self-propelled marble increased its mass by one-third during the course of its motion. The increase in mass followed by the marbles' heating is due to the adsorption of water vapor by their surface, which is permeable to gases. This gives rise to an exothermic chemical reaction, which

in turn gives rise to Marangoni thermo-capillary flow driving the marble. Thermo-physical analysis of the problem is presented. The role of soluto-capillary flow in self-propulsion is negligible.

\section{INTRODUCTION}

Development of active media exploiting chemical energy for their propulsion is of much interest in a view of a variety of applications, including micro- and biorobotics, ${ }^{1,2}$ drug delivery, $^{3-5}$ development of active colloids, ${ }^{6-8}$ and metamaterials $^{9}$ and microfluidics. ${ }^{10} \mathrm{~A}$ number of research groups demonstrated the potential of droplets as active media. ${ }^{11-16}$ Self-propulsion of nonstick droplets coated with colloidal particles, known also as liquid marbles, ${ }^{17-20}$ is of particular interest due to their high mobility, ${ }^{17}$ prolonged life span, ${ }^{21,22}$ and unusual quasi-elastic mechanical properties. ${ }^{18,23,24}$ Involving these properties already enabled attomole detection of toxins at the microliter scale, ${ }^{25}$ precise optical probing, ${ }^{26}$ development of smart lab-on-chip systems, ${ }^{10}$ living cell cultivation, ${ }^{27,28}$ and nonconventional computing. ${ }^{29,30}$ Actuating of liquid marbles by electric and magnetic fields ${ }^{31-34}$ and UV and IR irradiation ${ }^{35-37}$ was also reported. Liquid marbles placed on a liquid support may be effectively propelled by soluto-capillary ${ }^{38-40}$ and thermo-capillary Marangoni

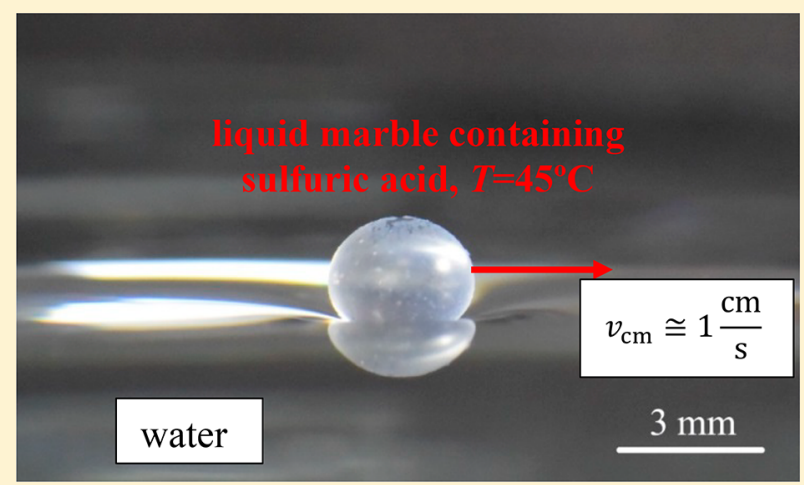

flows. ${ }^{41,42}$ In our present work, we demonstrate that liquid marbles containing sulfuric acid may be effectively displaced by thermo-capillary Marangoni flow. ${ }^{43-49}$ Self-propulsion took place under breaking of cylindrical symmetry of the water surface tension distribution in the vicinity of the marble contact area. Self-propulsion of liquid marbles is of particular interest in view of their potential in microfluidics enabling the handling of a small amount of fluid, thus opening new pathways in digital microfluidics. ${ }^{10}$

\section{EXPERIMENTAL METHODS}

Materials. Liquid marbles containing sulfuric acid were manufactured with extremely hydrophobic fumed fluorosilica powder. ${ }^{50}$ The primary diameter of the particles is $20-30 \mathrm{~nm}$ and they originate from hydrophilic silica (Wacker Chemie)

Received: June 27, 2018

Revised: July 24, 2018

Published: July 24, 2018 
after reaction with tridecafluoro-1,1,1,2-tetrahydrooctyltrimethoxysilane. $^{50}$ The residual silanol content on their surfaces is $50 \%$ and the fluorine content is $10.9 \%$. Sulfuric acid (ACS reagent, 95-98\%) was supplied by Sigma-Aldrich. The density and surface tension of sulfuric acid and its aqueous solution were extracted from ref 48. Deionized water was prepared from a synergy UV water purification system from Millipore SAS (France). The specific resistivity was $\rho=18.2$ $\mathrm{M} \Omega \mathrm{cm}$ at $25{ }^{\circ} \mathrm{C}$. Methyl orange and litmus paper were supplied by Merck.

Manufacture of Liquid Marbles. Droplets containing sulfuric acid were coated with fumed fluorosilica powder according to the protocol described in ref 36. Droplets of sulfuric acid of volume 5,10 , and $20 \mu \mathrm{L}$ were spread on a layer of fumed fluorosilica powder situated on a glass slide. The slide was vibrated slightly giving rise to the formation of liquid marbles. The marbles are not hermetically coated with powder and evaporate as discussed in detail in refs 21,22 , and36-38. The lifetime of marbles enabling observation of self-propulsion was established as ca. 1-2 min.

Registration of Self-Propulsion of Liquid Marbles. The marbles were placed on the surface of deionized water and floated, as shown in Figure 1. Their motion was registered

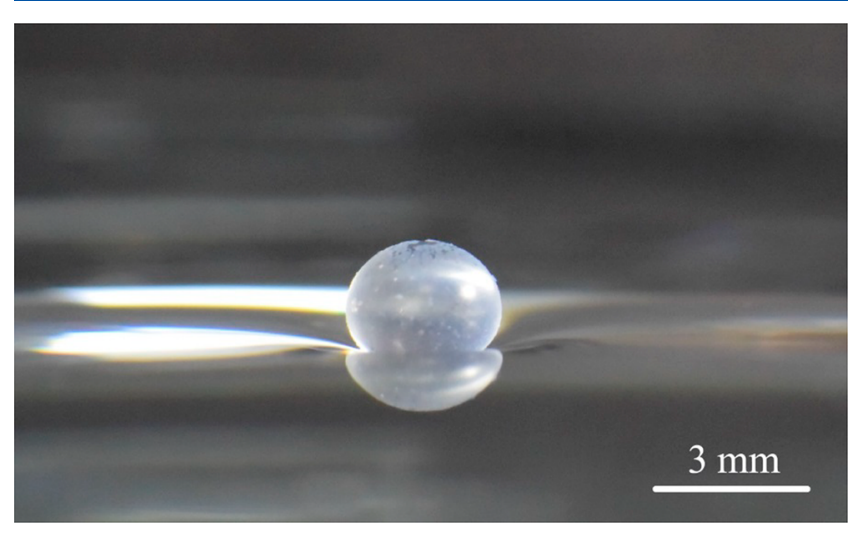

Figure 1. Liquid marble $(V=10 \mu \mathrm{L})$ filled with sulfuric acid floating on a water-air interface.

from above with a Therm-App TAS19AQ-1000-HZ thermal camera. All the experiments were performed around 23-25 ${ }^{\circ} \mathrm{C}$. The relative humidity $(\mathrm{RH})$ was $65 \pm 5 \%$.

Thermal Imaging of Self-Propulsion. Thermal imaging of the self-propulsion was monitored with a Therm-App TAS19AQ-1000-HZ thermal camera (Opgal Optronic Industries, Karmiel, Israel) equipped with a long wavelength infrared $6.8 \mathrm{~mm} \mathrm{f} / 1.4$ lens (see Supporting Video 1). The temperatures of the water surface and of the surface of the marbles were captured. The resolution, accuracy, sensitivity and the frame rate of the camera were $384 \times 288$ pixels (>110 000 pixels), \pm $3{ }^{\circ} \mathrm{C}$ (or $3 \%$ under $25{ }^{\circ} \mathrm{C}$ ), NEDT (noise equivalent differential temperature $<0.07{ }^{\circ} \mathrm{C}$ and 26 frames $/ \mathrm{s}$, respectively.

Measurement of the Mass Increase. Marbles containing sulfuric acid increased their mass as a result of water adsorption. The growth of the marbles' mass was measured in the following model experiment: marbles with an initial volume of $20 \mu \mathrm{L}$ containing sulfuric acid were kept at rest in the nearest possible vicinity to the water/vapor interface. The mass of a marble was measured with a four decimal place analytical balance ASB-310-C2 until their bursting.

\section{RESULTS AND DISCUSSION}

Self-propulsion of liquid marbles containing neat sulfuric acid placed on a water-vapor interface was observed for various marble volumes $(V=5-20 \mu \mathrm{L})$. The liquid marble containing sulfuric acid of volume $10 \mu \mathrm{L}$ floating on the water surface is depicted in Figure 1. The typical duration of the self-propelled motion $\tau_{\text {motion }}$ was between 60 and $120 \mathrm{~s}$. Typical time dependencies of the velocity of the center of mass of the marbles are depicted in Figure 2. The characteristic velocities

(a)

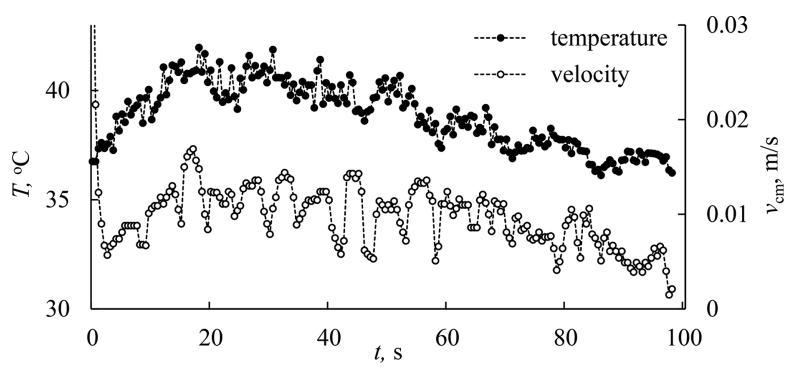

(b)

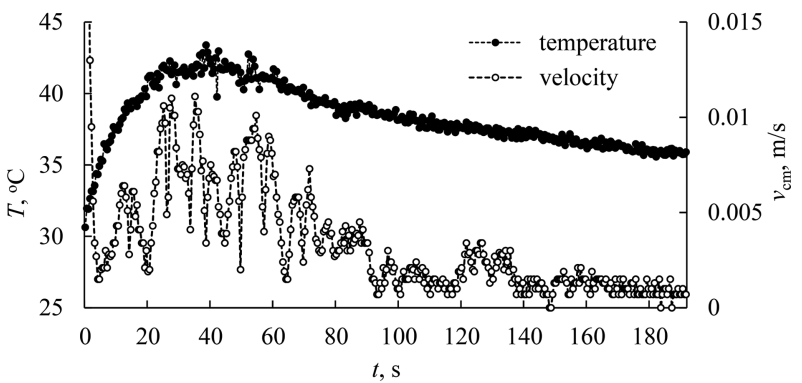

(c)

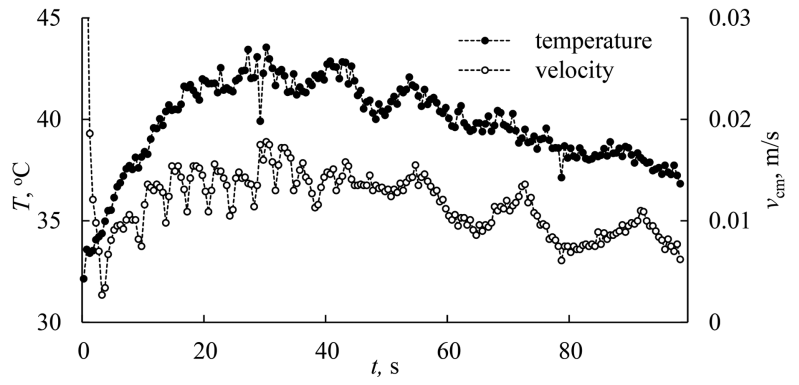

Figure 2. Time dependencies of the temperature measured at the marble surface and the velocity of the center of mass of liquid marbles for various marble volumes $V$ : (a) 5, (b) 10 , and (c) $20 \mu \mathrm{L}$.

of the self-propulsion are of the order of $v_{\mathrm{cm}} \sim 0.01 \mathrm{~m} / \mathrm{s}$. The oscillations of the velocity of the center of mass and the temperature of the marbles with a characteristic period of $5 \mathrm{~s}$ seen in Figure 2 originate from their bouncing off the water meniscus, adjacent to the glass walls of the Petri dish. The marbles moved at first by inertia, reflected off the Petri dish rim and then stopped in the vicinity of the center of the Petri dish. There they were at rest, and they then started their selfpropelled motion after ca. 1-10 s delay (see the Supporting Video 1). This delay is of great importance for the study of self-propulsion owing to the fact that marbles "forgot" their initial inertial motion and started the self-propelled motion from rest.

It is important to note that the marbles containing sulfuric acid were markedly heated when placed on a water surface as can be seen from Figures 2 and 3. The maximum surface temperature of $70{ }^{\circ} \mathrm{C}$ was registered for the marbles at rest. The surface temperature of the marbles decreased to $40-50$ 


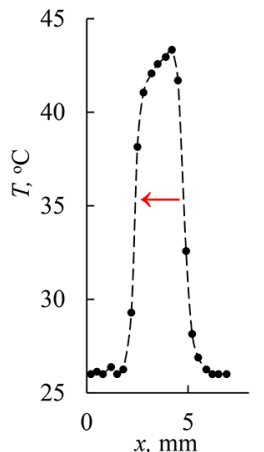

(a)

Figure 3. Profiles of temperature $(a, b, c)$ registered across the lateral equatorial section of a liquid marble (taken at the top part of the marble) in the direction of motion (red arrow). The graphs represent different marbles of the same volume $V=10 \mu \mathrm{L}$.

${ }^{\circ} \mathrm{C}$ in the course of their self-propelled motion, as shown in Figures 2 and 3 . The thermal contrast between the planar water surface and the surface of the marbles of $20-25^{\circ} \mathrm{C}$ was registered. It is reasonable to explain heating of the marbles by the exothermic reaction between water and sulfuric acid which takes place when the liquid marbles absorb water vapor, as shown schematically in Figure 4 (remember that the liquid marbles are not coated hermetically with fumed fluorosilica particles $^{38-40}$ ).

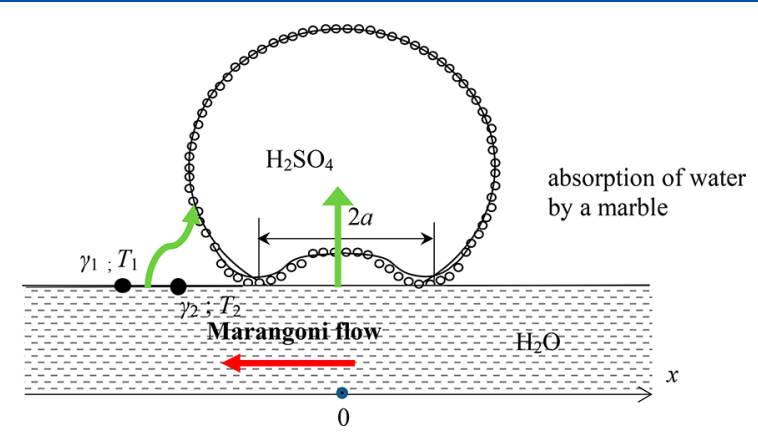

Figure 4. Marble moves in the direction denoted $(-x)$ which coincides with the direction of Marangoni flow, marked by the red arrow. Green arrows illustrate evaporation of water followed by adsorption of water molecules by the marbles surface, resulting in inhomogeneous heating of a marble giving rise to the thermo-capillary Marangoni flow; the inter-relationships $T_{1}<T_{2}, \gamma_{2}<\gamma_{1}$ take place.

The intensive absorption of water by liquid marbles at rest is illustrated in Figure 5, demonstrating the increase of the diameter and the mass $(m)$ of sulfuric acid-filled marbles with time, $t$. The initial stage of the mass growth of sulfuric acid marbles is well described by the linear time dependence:

$$
m=m_{0}+\alpha t ; \alpha \cong 8 \times 10^{-4} \frac{\mathrm{g}}{\mathrm{s}}
$$

where $m_{0}$ is the initial mass. The increase in the mass growth of marbles in model experiments (performed with marbles in rest) lasts continuously for the time span $\tau_{\text {mass }}^{\text {growth }} \approx 10^{3} \mathrm{~s}$. Thus, the inter-relation $\tau_{\text {mass }}^{\text {growth }} \gg \tau_{\text {motion }}$ takes place. This means that the marble increases its mass continuously over the course of its motion. The self-propelled marble increased its mass by one-third during its entire displacement. Cracks in the powder coating of marbles were registered after ca. $10^{3} \mathrm{~s}$ of the mass
A

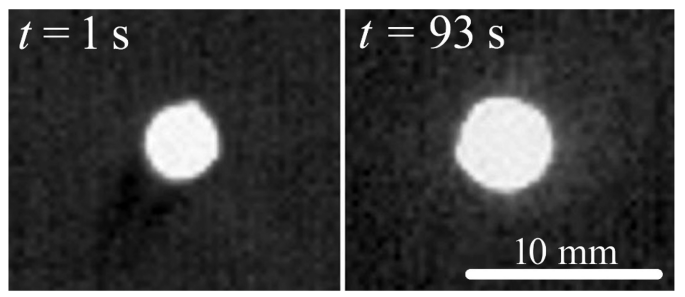

B

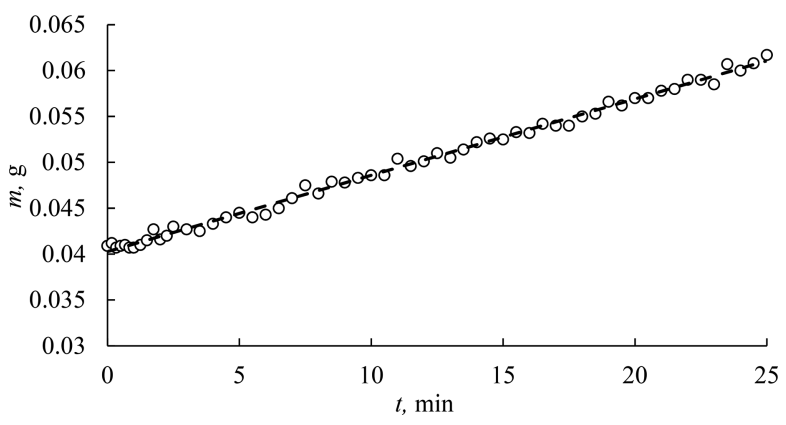

Figure 5. Time dependence of (A) size and (B) mass of a liquid marble (initial volume $V=20 \mu \mathrm{L}$ ) containing sulfuric acid.

increase, followed by bursting of marbles. This time span restricted the lifetime and consequently the time span of the self-propulsion.

Now inspect qualitatively the kinematics of the selfpropulsion. The marbles containing sulfuric acid demonstrated a kind of self-propulsion similar but somewhat different from that described in refs 38 and 39, in which self-propulsion of marbles filled with an aqueous solution of alcohol was studied. Marbles filled with sulfuric acid moved markedly slower than those containing aqueous alcohol; the maximum velocity of their center of mass was at least 1 order of magnitude smaller than in the latter case. ${ }^{38,39}$ However, the characteristic velocity of the center of mass of marbles was close to that registered for the translational motion of liquid marbles filled with aqueous solutions of camphor. ${ }^{40}$ This means that gradients of water surface tension driving marbles containing aqueous solutions of alcohols are markedly larger than those inherent for the marbles filled with aqueous solutions of camphor and sulfuric acid. Recall that the fumed fluorosilica particles were used for all of the aforementioned marbles as a coating powder. ${ }^{38,40}$

The time taken for marbles to attain a more or less uniform averaged velocity motion (in other words the characteristic time of their acceleration) was established experimentally as $\tau_{\text {acc }} \cong 20 \mathrm{~s}$ (see Figure 2). It seems plausible to ascribe this time span to the viscous (skin) dissipation. ${ }^{38,51}$ Indeed, in our experiments the characteristic velocity of the center mass of marbles was approximately $v_{\mathrm{cm}} \sim 0.01 \mathrm{~m} / \mathrm{s}$ and it was at least an order of magnitude smaller than $c_{\min }=\sqrt[4]{\frac{4 g \gamma}{\rho}} \cong 0.23 \frac{\mathrm{m}}{\mathrm{s}}$ (as calculated for water), i.e. the threshold velocity at which the wave drag effects become important. ${ }^{51}$ The characteristic time necessary for establishing the thermal equilibrium within a marble is supplied by $\tau_{\text {therm }} \cong \frac{L^{2}}{\alpha}$, where $L$ is the characteristic dimension of a marble and $\alpha$ is the thermal diffusivity of sulfuric acid. Assuming $L \cong 1.5 \mathrm{~mm}$ (which is the order of magnitude of the contact radius of a marble ${ }^{39}$ ) and 
$\alpha \cong 10^{-7} \frac{\mathrm{m}^{2}}{\mathrm{~s}}$ (see refs 52 and 53,) yields $\tau_{\text {therm }} \cong 20 \mathrm{~s}$. The relatively high values of $\tau_{\text {therm }}$ (actually responsible for creating the gradient of surface tension driving the marble) are due to the low values of thermal diffusivity of the sulfuric acid. ${ }^{52}$

The characteristic time of viscous dissipation may be roughly estimated as $\tau_{\text {visc }} \cong \frac{L^{2}}{\nu}$, where $\simeq \cong 10^{-6} \frac{\mathrm{m}^{2}}{\mathrm{~s}}$ is the kinematic viscosity of water. We obtain $\tau_{\text {visc }} \cong 2.5 \mathrm{~s}$, which is smaller than the experimentally established time of the marbles' acceleration. It is reasonable to ascribe this discrepancy to the continuously increasing mass of a marble over the course of its motion. Anyway, the following hierarchy of time scales is inherent for the self-propulsion of liquid marbles filled with sulfuric acid:

$$
\tau_{\text {mass }}^{\text {growth }} \cong \tau_{\text {motion }}>\tau_{\text {therm }} \cong \tau_{\text {acc }}>\tau_{\text {visc }}
$$

This hierarchy of time scales defines the character of the observed self-propulsion. Marbles continuously absorb the "water fuel" from the water-vapor interface, which heats them as a result of the exothermic chemical reaction. The time span necessary to equating a temperature within a marble is as large as ca. $20 \mathrm{~s}$, thus providing sufficient time to accelerate a marble by the Marangoni thermo-capillary flow. During approximately $20 \mathrm{~s}$ marbles come to approximately uniform velocity, defined by the balance of the Marangoni-inspired force and viscous dissipation. It is instructive to analyze qualitatively the mechanism of self-propulsion of liquid marbles. First, consider the origin of the thermo-capillary Marangoni flow. Breaking of the cylindrical symmetry of the thermal field created on the surface of a water support, shown in Figure 6, arises from various factors, namely: (i) nonuniform heating of marbles depicted in Figure 3 and (ii) translational motion of a marble.

Consider first the initial stage of the self-propulsion of a liquid marble. Immediately after being placed on the water surface, a marble has been at rest for ca. 1-10 s, and the nonhomogeneous thermal field forms around the marble, as shown in Figure 6A. The temperature jump across this field is about $1 \mathrm{~K}$, as shown in Figure 6B and Figure 7. This jump may be related to the inhomogeneity of the temperature of the marble itself, illustrated in Figure 3.

The meaningful physical question is what kind of Marangoni flow (thermo- or soluto-capillary) plays the decisive role in the triggering of the self-propulsion? Generally, soluto-capillary Marangoni flows may give rise to self-propulsion of marbles when alcohols or camphor evaporate from them, breaking the symmetry of the experimental situation as reported in refs $36-38$. The surface tension of sulfuric acid is lower than that of water $\left(\gamma_{\mathrm{H}_{2} \mathrm{SO}_{4}} \cong 55 \frac{\mathrm{mJ}}{\mathrm{m}^{2}}\right) ; 3$ hence, its adsorption by the water surface may promote Marangoni flow. However, sulfuric acid is not a surfactant and it decreases the surface tension of water only negligibly. We measured this decrease experimentally as described in ref 54 and did not record any change of the surface tension at the water-vapor interface (within $\pm 0.1 \frac{\mathrm{mJ}}{\mathrm{m}^{2}}$ ). In order to check the true role of evaporation of sulfuric acid from marbles we performed the following experiment: marbles were placed on litmus paper and aqueous solutions of methyl orange $(c=0.03$ wt \%) as shown in Figure 8 (see Supporting Video 2). No evidence of evaporation of sulfuric acid from marbles was registered. We conclude that asymmetrical heating of the water-vapor interface sets the liquid marble in motion in the $(-x)$ direction, as shown schematically in Figure 4 (the
A

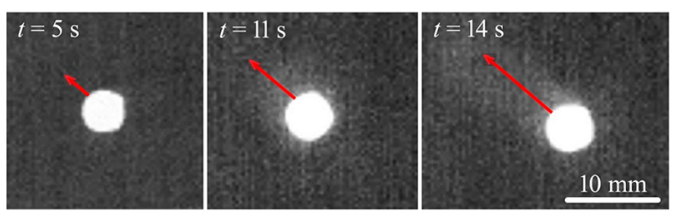

B

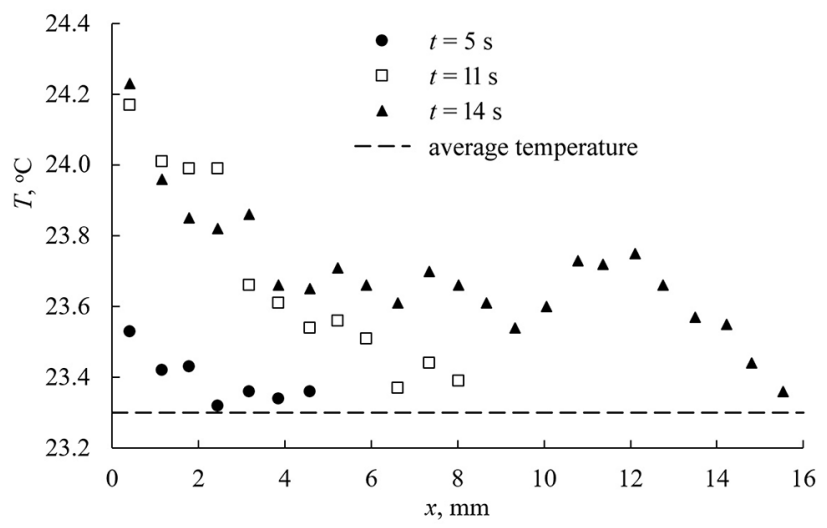

Figure 6. Breaking symmetry of temperature field in the vicinity of a sulfuric acid-filled marble $(V=20 \mu \mathrm{L})$. The marbles moved in the direction denoted $(-x)$ (see Figure 4). (A) Sequence of thermographic images of thermal fields taken in the course of motion of the marble (from rest to movement). Red arrows indicate the direction, denoted $x$ (opposite to the marble's motion) at which the temperature at the water-vapor interface was measured. (B) Temperature profiles along the direction $x$ are presented.

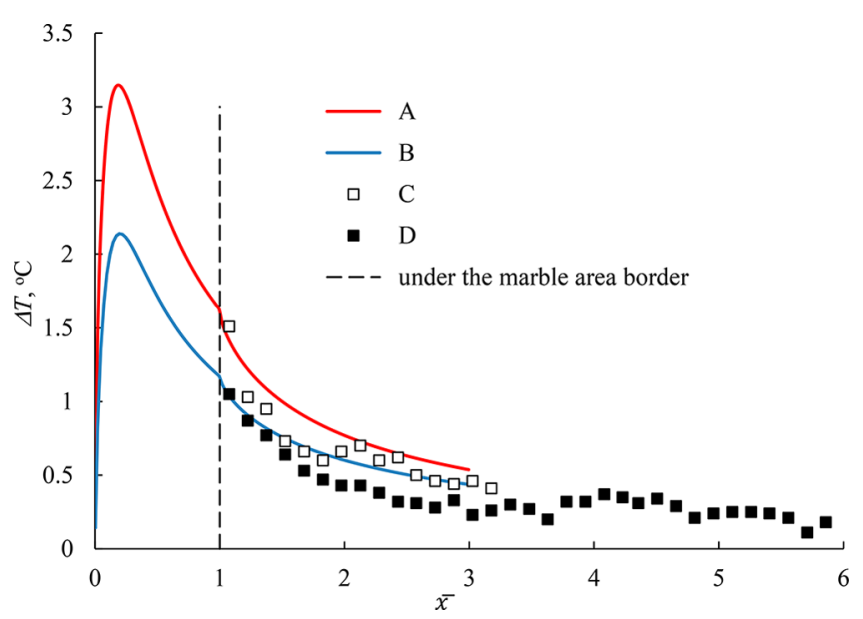

Figure 7. Comparison of the measured temperature profiles across the water/vapor interface with those calculated with thermophysical model (see the Supporting Information). Curves A and B represent the calculated curves; $\Delta T(\bar{x})$ denotes superheat, calculated relative to background temperature of the water/vapor interface; $\bar{x}$ is the dimensionless distance $\bar{x}=\frac{x}{R}$ (0 represents the coordinate of marble front point); curves $\mathrm{C}$ and $\mathrm{D}$ depict the values of $\Delta T$ measured in the thermal tail (see Figure 6A). The parameters of the model are droplet radius $R=2 \mathrm{~mm}$, clearance $d=25 \mu \mathrm{m}$, velocity of the center of mass $v_{\mathrm{cm}}=4 \frac{\mathrm{mm}}{\mathrm{s}}$ for curve A; $v_{\mathrm{cm}}=10 \frac{\mathrm{mm}}{\mathrm{s}}$ for curve B. The measured temperatures are presented for the marble with volume $V=20 \mu \mathrm{L}$ taken $94 \mathrm{~s}$ (curve C) and $28 \mathrm{~s}$ (curve D) after the onset of the selfpropelled motion. The velocities of the center of mass of marbles are $v_{c m}=3.5 \frac{\mathrm{mm}}{\mathrm{s}}$ (for curve C) and $v_{c m}=10.05 \frac{\mathrm{mm}}{\mathrm{s}}$ (for curve D). 

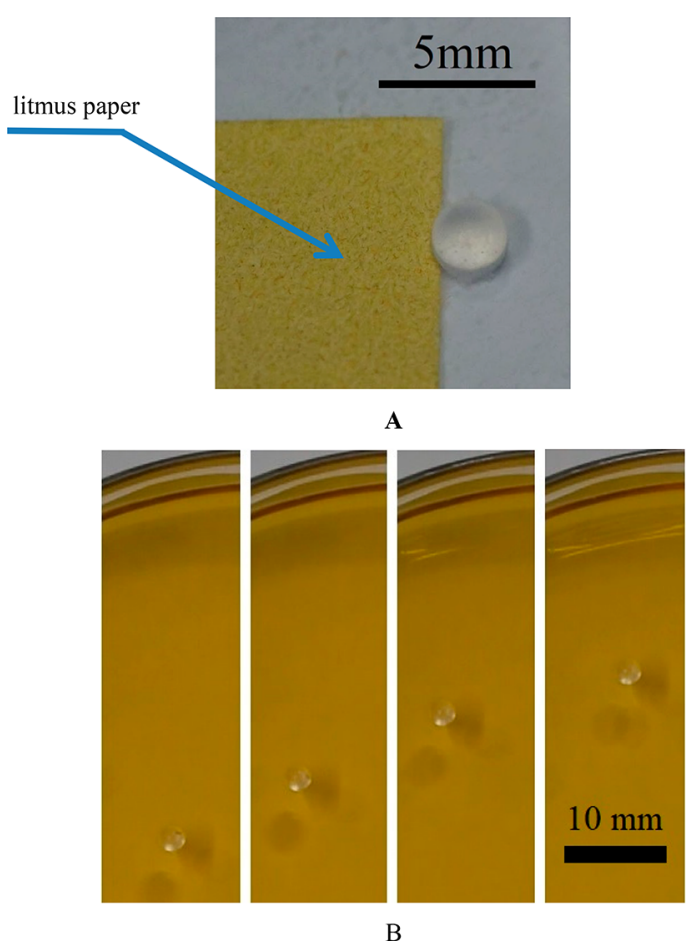

Figure 8. Testing of the role of evaporation of sulfuric acid from the liquid marble is shown. (A) Liquid marble containing sulfuric acid placed in the vicinity of the litmus paper is depicted. (B) Sequence of images demonstrating self-propulsion of a liquid marble filled with sulfuric acid over the surface of an aqueous solution of methyl orange $(c=0.03 \mathrm{wt} \%)$ is shown. The time span between images was $4 \mathrm{~s}$.

reference point coincides with the axis of symmetry of the marble).

Assume that the observed self-propulsion is due to the thermo-capillary Marangoni flow. Thus, the characteristic velocity of the marble may be roughly estimated as

$$
v_{c m} \cong \frac{1}{\chi} \frac{\Delta \gamma}{\eta_{w}} \cong \frac{1}{\chi} \frac{\left|\frac{\partial \gamma}{\partial T}\right| \Delta T}{\eta_{w}}
$$

where $\Delta \gamma$ is the jump in the surface tension driving the selfpropulsion due to the thermo-capillarity, $\eta_{\mathrm{w}} \cong 9 \times 10^{-4} \mathrm{~Pa} \times \mathrm{s}$ is the dynamic viscosity of water at ambient conditions, $\chi \cong 10$ is the dimensionless coefficient introduced in ref $39, \Delta T \cong 1 \mathrm{~K}$ is the temperature jump across the water surface and $\left|\frac{\partial \gamma}{\partial T}\right| \cong 17.7 \times 10^{-5} \frac{\mathrm{J}}{\mathrm{K} \times \mathrm{m}^{2}}$ is the modulus of the surface tension gradient of water (see ref 52). The calculations according to eq 3 yield the estimation $v_{\mathrm{cm}} \cong 0.02 \frac{\mathrm{m}}{\mathrm{s}}$ in satisfactory agreement with the experimental data supplied in Figure 2.

After the onset of self-propulsion, the breaking of the thermal field symmetry is due to the preferred direction of the marble, as demonstrated in the Supporting Information, representing thorough thermo-physical analysis of the problem. The comparison of thermal fields established experimentally with thermal imaging and those calculated with the model are depicted in Figure 7. The satisfactory coincidence of the experimental data with the predictions of the model is recognized. Formation and spreading of the thermal trace formed on the water surface by a self-propelled liquid marble calculated according to a model and depicted in
Figures S1 and S2 are close to the experimental findings both qualitatively and quantitatively.

Now consider the cyclic character of the motion of a marble, illustrated in Figure 2. When a marble is stopped due to the friction of bouncing from the rim of the Petri dish, the decrease in its velocity results in considerable additional asymmetrical heating of the water surface triggering a new cycle of selfpropulsion, as demonstrated in the Supporting Information and shown in Figure S3. Note that the displacement of marbles in turn enhances the water evaporation within the layer separating the marble from the supporting liquid and thus also promoting the motion. ${ }^{38}$ The complicated geometry of the vapor layer, separating a marble from water and depicted schematically in Figure 4, was treated in detail in ref 55. However, for the sake of simplicity we adopted that the clearance between a marble and liquid support was constant in a course of self-propulsion (see the Supporting Information presenting the thermo-physical model).

\section{CONCLUSIONS}

Liquid marbles are droplets coated with hydrophobic powders. ${ }^{18-20,49}$ The permeability of the solid coating to gases supplies to liquid marbles a diversity of useful properties enabling gas sensing ${ }^{56,57}$ and carrying out microchemical reactions and cell interactions. ${ }^{58,59}$ Among other effects, connection of volatile liquids, filling marbles and exposure to the atmosphere provides their self-propulsion when placed on a liquid support. ${ }^{38-40}$ We report here self-propulsion of liquid marbles $(V=5-20 \mu \mathrm{L})$ filled with sulfuric acid and coated with very hydrophobic fumed fluorosilica powder, floating at ambient conditions on a water surface. Floating marbles containing sulfuric acid were heated to $70{ }^{\circ} \mathrm{C}$ (at rest) when placed on water due to the exothermic chemical reaction arising under adsoption of water vapor by the surface of the marble. Thermal imaging evidenced nonuniform heating of marbles, breaking the spherical symmetry of the experimental situation and giving rise to the Marangoni thermo-capillary flow, moving marbles with the velocity of the center of mass of ca. $v_{\mathrm{cm}} \cong 0.01 \frac{\mathrm{m}}{\mathrm{s}}$ and continuing for a couple of minutes. Selfpropulsion is triggered by the nonuniform thermal field created within the marble which is due to asymmetric absorption of water by a marble. A nonuniform thermal field was observed on the water-vapor interface over the course of selfpropulsion. The thermo-physical analysis of the self-propulsion is reported, explaining the origin of the nonsymmetrical thermal field on the surface of water, resulting in Marangoni thermo-capillary flows.

\section{ASSOCIATED CONTENT}

\section{Supporting Information}

The Supporting Information is available free of charge on the ACS Publications website at DOI: 10.1021/acs.jpcb.8b06136.

Thermo-physical analysis of self-propulsion (PDF)

Supporting Video 1: Movie demonstrating self-propulsion of the $20 \mu \mathrm{L}$ liquid marble filled with sulfuric acid (AVI)

Supporting Video 2: Movie demonstrating self-propulsion of $10 \mu \mathrm{L}$ liquid marbles containing sulfuric acid taking place on the surface of aqueous solution of methyl orange (AVI) 


\section{AUTHOR INFORMATION}

\section{Corresponding Author}

*(E.B.) E-mail: edward@ariel.ac.il. Telephone: +972-3-9066134. Fax: +972-3-906-6621.

\section{ORCID $\odot$}

Bernard P. Binks: 0000-0003-3639-8041

Edward Bormashenko: 0000-0003-1356-2486

\section{Notes}

The authors declare no competing financial interest.

\section{ACKNOWLEDGMENTS}

Acknowledgement is made to the donors of the Israel Ministry of Absorption for the partial support of the scientific activity of M.F. The authors are thankful to anonymous reviewers for their instructive reviewing of the manuscript.

\section{REFERENCES}

(1) Čejková, J.; Banno, T.; Hanczyc, M. M.; Štěpánek, F. Droplets as Liquid Robots. Artificial Life 2017, 23, 528-549.

(2) Lauder, G. V.; Anderson, E. J.; Tangorra, J.; Madden, P. G. A. Fish biorobotics: Kinematics and Hydrodynamics of Self-Propulsion. J. Exp. Biol. 2007, 210, 2767-2780.

(3) Esteban-Fernández de Ávila, B.; Angsantikul, P.; Li, J.; LopezRamirez, M. A.; Ramírez-Herrera, D. E.; Thamphiwatana, S.; Chen, C.; Delezuk, J.; Samakapiruk, R.; Ramez, V.; et al. MicromotorEnabled Active Drug Delivery for in vivo Treatment of Stomach Infection. Nat. Commun. 2017, 8, 272.

(4) Wu, Z.; Wu, Y.; He, W.; Lin, X.; Sun, J.; He, Q. Self-Propelled Polymer-Based Multilayer Nanorockets for Transportation and Drug Release. Angew. Chem., Int. Ed. 2013, 52, 7000-7003.

(5) Gao, W.; Wang, J. Synthetic Micro/Nanomotors in Drug Delivery. Nanoscale 2014, 6, 10486-10494.

(6) Di Leonardo, R. Controlled Collective Motions. Nat. Mater. 2016, 15, 1057-1058.

(7) Zöttl, A.; Stark, H. Hydrodynamics Determines Collective Motion and Phase Behavior of Active Colloids in Quasi-TwoDimensional Confinement. Phys. Rev. Lett. 2014, 112, 118101.

(8) Yan, J.; Han, M.; Zhang, J.; Xu, C.; Luijten, E.; Granick, S. Reconfiguring Active Particles by Electrostatic Imbalance. Nat. Mater. 2016, 15, 1095-1100.

(9) Alù, A. Topological Order Gets Active. Nat. Phys. 2017, 13, 1038-1039.

(10) Nguyen, N-Tr.; Hejazian, M.; Ooi, Vh. H.; Kashaninejad, N. Recent Advances and Future Perspectives on Microfluidic Liquid Handling. Micromachines 2017, 8, 186.

(11) Izri, Z.; van der Linden, M. N.; Michelin, S.; Dauchot, O. SelfPropulsion of Pure Water Droplets by Spontaneous MarangoniStress-Driven Motion. Phys. Rev. Lett. 2014, 113, 248302.

(12) Janssens, S. D.; Koizumi, S.; Fried, E. Behavior of Self-Propelled Acetone Droplets in a Leidenfrost State on Liquid Substrates. Phys. Fluids 2017, 29, 032103.

(13) Chen, Y.-J.; Nagamine, Y.; Yoshikawa, K. Self-Propelled Motion of a Droplet Induced by Marangoni-Driven Spreading. Phys. Rev. E 2009, 80, 016303.

(14) Yabunaka, S.; Ohta, T.; Yoshinaga, N. Self-Propelled Motion of a Fluid Droplet under Chemical Reaction. J. Chem. Phys. 2012, 136, 074904.

(15) Kuhn, P. T.; De Miranda, B. S.; van Rijn, P. Directed Autonomic Flow: Functional Motility Fluidics. Directed Autonomic Flow: Functional Motility Fluidics. Adv. Mater. 2015, 27, 7401-7406.

(16) Li, J.; Hou, Y.; Liu, Y.; Hao, C.; Li, M.; Chaudhury, M. K.; Yao, Sh.; Wang, Z. Directional Transport of High-Temperature Janus Droplets Mediated by Structural Topography. Nat. Phys. 2016, 12, 606-612.

(17) Aussillous, P.; Quéré, D. Liquid Marbles. Nature 2001, 411, 924-927.
(18) Bormashenko, E. Liquid Marbles, Elastic Nonstick Droplets: From Mini-Reactors to Self-Propulsion. Langmuir 2017, 33, 663-669.

(19) Binks, B. P.; Murakami, R. Phase Inversion of ParticleStabilized Materials from Foams to Dry Water. Nat. Mater. 2006, 5, 865-869.

(20) McHale, G.; Newton, M. I. Liquid Marbles: Principles and Applications. Soft Matter 2011, 7, 5473-5481.

(21) Tosun, A.; Erbil, H. Y. Evaporation Rate of PTFE Liquid Marbles. Appl. Surf. Sci. 2009, 256, 1278-1283.

(22) Dandan, M.; Erbil, H. Y. Evaporation Rate of Graphite Liquid Marbles: Comparison with Water Droplets. Langmuir 2009, 25, 8362-8367.

(23) Asare-Asher, S.; Connor, J. N.; Sedev, R. Elasticity of Liquid Marbles. J. Colloid Interface Sci. 2015, 449, 341-346.

(24) Rendos, A.; Alsharif, N.; Kim, B. L.; Brown, K. A. Elasticity and Failure of Liquid Marbles: Influence of Particle Coating and Marble Volume. Soft Matter 2017, 13, 8903-8909.

(25) Han, X.; Koh, C.; Lee, H. K.; Chew, W. S.; Ling, X. Y. Microchemical Plant in a Liquid Droplet: Plasmonic Liquid Marble for Sequential Reactions and Attomole Detection of Toxin at Microliter Scale. ACS Appl. Mater. Interfaces 2017, 9, 39635-39640.

(26) Zhao, Y.; Xu, Z.; Parhizkar, M.; Fang, J.; Wang, X.; Lin, T. Magnetic Liquid Marbles, their Manipulation and Application in Optical Probing. Microfluid. Nanofluid. 2012, 13, 555-564.

(27) Li, H.; Liu, P.; Kaur, G.; Yao, X.; Yang, M. Transparent and Gas-Permeable Liquid Marbles for Culturing and Drug Sensitivity Test of Tumor Spheroids. Adv. Healthcare Mater. 2017, 6, 1700185.

(28) Vadivelu, R.; Kamble, H.; Munaz, A.; Nguyen, N.-T. Liquid Marble as Bioreactor for Engineering Three-Dimensional Toroid Tissues. Sci. Rep. 2017, 7, 12388.

(29) Fullarton, C.; Draper, Th. C.; Phillips, N.; Mayne, R.; de Lacy Costello, B. P. J.; Adamatzky, A. Evaporation, Lifetime, and Robustness Studies of Liquid Marbles for Collision-Based Computing. Langmuir 2018, 34, 2573-2580.

(30) Draper, T. C.; Fullarton, C.; Phillips, N.; de Lacy Costello, B. P. J.; Adamatzky, A. Liquid Marble Interaction Gate for Collision-Based Computing. Mater. Today 2017, 20, 561-568.

(31) Bormashenko, E.; Pogreb, R.; Stein, T.; Whyman, G.; Schiffer, M.; Aurbach, D. Electrically Deformable Liquid Marbles. J. Adhes. Sci. Technol. 2011, 25, 1371-1377.

(32) Bormashenko, E.; Pogreb, R.; Bormashenko, Y.; Musin, A.; Stein, T. New Investigations on Ferrofluidics: Ferrofluidic Marbles and Magnetic-Field-Driven Drops on Superhydrophobic Surfaces. Langmuir 2008, 24, 12119-12122.

(33) Khaw, M. K.; Ooi, Ch. H.; Mohd-Yasin, F.; Nguyen, A. V.; Evans, G. M.; Nguyen, N.-Tr. Dynamic Behaviour of a Magnetically Actuated Floating Liquid Marble. Microfluid. Nanofluid. 2017, 21, 110.

(34) Vialetto, J.; Hayakawa, M.; Kavokine, N.; Takinoue, M.; Varanakkottu, S. N.; Rudiuk, S.; Anyfantakis, M.; Morel, M.; Baigl, D. Magnetic Actuation of Discrete Liquid Entities with a Deformable Paramagnetic Liquid Substrate. Angew. Chem., Int. Ed. 2017, 56, $16565-16570$

(35) Kavokine, N.; Anyfantakis, M.; Morel, M.; Rudiuk, S.; Bickel, Th.; Baigl, D. Light-Driven Transport of a Liquid Marble with and Against Surface Flows. Angew. Chem. 2016, 128, 11349-11353.

(36) Paven, M.; Mayama, K.; Sekido, Y.; Butt, H.-J.; Nakamura, Y.; Fujii, S. Light-Driven Delivery and Release of Materials Using Liquid Marbles. Adv. Funct. Mater. 2016, 26, 3199-3206.

(37) Fujii, S.; Yusa, Sh-I.; Nakamura, Y. Stimuli-Responsive Liquid Marbles: Controlling Structure, Shape, Stability, and Motion. Adv. Funct. Mater. 2016, 26, 7206-7223.

(38) Bormashenko, E.; Bormashenko, Y.; Grynyov, R.; Aharoni, H.; Whyman, G.; Binks, B. P. Self-Propulsion of Liquid Marbles: Leidenfrost-Like Levitation Driven by Marangoni Flow. J. Phys. Chem. C 2015, 119, 9910-9915.

(39) Ooi, Ch. H.; van Nguyen, A.; Evans, G. M.; Gendelman, O.; Bormashenko, E.; Nguyen, N.-Tr A Floating Self-Propelling Liquid Marble Containing Aqueous Ethanol Solutions. RSC Adv. 2015, 5, 101006-101012. 
(40) Bormashenko, E.; Frenkel, M.; Bormashenko, Y.; Chaniel, G.; Valtsifer, V.; Binks, B. P. Superposition of Translational and Rotational Motions Under Self-Propulsion of Liquid Marbles Filled by Aqueous Solutions of Camphor. Langmuir 2017, 33, 1323413241.

(41) Kawashima, H.; Mayama, H.; Nakamura, Y.; Fujii, S. Hydrophobic Polypyrroles Synthesized by Aqueous Chemical Oxidative Polymerization and Their Use as Light-Responsive Liquid Marble Stabilizers. Polym. Chem. 2017, 8, 2609-2618.

(42) Kawashima, H.; Paven, M.; Mayama, H.; Butt, H.-J.; Nakamura, Y.; Fujii, S. Transfer of Materials from Water to Solid Surfaces Using Liquid Marbles. ACS Appl. Mater. Interfaces 2017, 9, 33351-33359.

(43) Nepomnyashchy, A. A.; Velarde, M. G.; Colinet, P. Interfacial Phenomena and Convection; Chapman \& Hall/CRC Press: Boca Raton, FL, 2002.

(44) Bormashenko, E. Physics of Wetting; de Gruyter: Berlin, 2017.

(45) Krechetnikov, R. Thermodynamics of Chemical MarangoniDriven Engines. Soft Matter 2017, 13, 4931-4950.

(46) Colinet, P.; Legros, J. C.; Velarde, M. G. Nonlinear Dynamics of Surface-Tension-Driven Instabilities; Wiley: Berlin, 2001.

(47) de Gennes, P. G.; Brochard-Wyart, F.; Quéré, D. Capillarity and Wetting Phenomena; Springer: Berlin, 2003.

(48) Venerus, D. C.; Nieto Simavilla, D. Tears of Wine: New Insights on an Old Phenomenon. Sci. Rep. 2015, 5, 16162.

(49) Ryazantsev, Y. S.; Velarde, M. G.; Rubio, R. G.; Guzmán, E.; Ortega, F.; López, P. Thermo- and Soluto-Capillarity: Passive and Active Drops. Adv. Colloid Interface Sci. 2017, 247, 52-80.

(50) Binks, B. P.; Tyowua, A. T. Influence of the Degree of Fluorination on the Behavior of Silica Particles at Air-Oil Surfaces. Soft Matter 2013, 9, 834-845.

(51) Le Merrer, M.; Clanet, C.; Quere, D.; Raphael, E.; Chevy, F. Wave Drag on Floating Bodies. Proc. Natl. Acad. Sci. U. S. A. 2011, 108, 15064-15068.

(52) CRC Handbook of Chemistry and Physics, 91st ed.; Haynes, W. M., Ed.; CRC Press: Boca Raton, FL, 2010.

(53) Myhre, C. E. L.; Nielsen, C. J.; Saastad, O. W. Density and Surface Tension of Aqueous $\mathrm{H}_{2} \mathrm{SO}_{4}$ at Low Temperature. J. Chem. Eng. Data 1998, 43, 617-622.

(54) Musin, A.; Grynyov, G.; Frenkel, M.; Bormashenko, E. SelfPropulsion of a Metallic Superoleophobic Micro-Boat. J. Colloid Interface Sci. 2016, 479, 182-188.

(55) Burton, J. C.; Sharpe, A. L.; van der Veen, R. C.; Franco, A.; Nagel, S. R. The Geometry of the Vapor Layer Under a Leidenfrost Drop. Phys. Rev. Lett. 2012, 109, 074301.

(56) Tian, J.; Arbatan, T.; Li, X.; Shen, W. Liquid Marble for Gas Sensing. Chem. Commun. 2010, 46, 4734-4736.

(57) Tian, J.; Arbatan, T.; Li, X.; Shen, W. Porous Liquid Marble Shell Offers Possibilities for Gas Detection and Gas Reactions. Chem. Eng. J. 2010, 165, 347-353.

(58) Xue, Y.; Wang, H.; Zhao, Y.; Dai, L.; Feng, L.; Wang, X.; et al. Magnetic Liquid Marbles: A "Precise" Miniature Reactor. Adv. Mater. 2010, 22, 4814-4818.

(59) Vadivelu, R. K.; Kamble, H.; Munaz, A.; Nguyen, N.-Tr. Liquid Marbles as Bioreactors for the Study of Three-Dimensional Cell Interactions. Biomed. Microdevices 2017, 19, 31. 